Submitted on May 2, 2020.

\title{
Polyorganosilazanes: production, properties, application
}

\author{
(C) Andrey M. Kontorov, ${ }^{*+}$ and Alexander Yu. Glushchenko \\ "Rus Injection”. Saltykovskaya St., House 37, Building 1, Room 1. Moscow, 111672. Russia. \\ E-mail:ankont2@yandex.ru
}

\author{
*Supervising author; ${ }^{+}$Corresponding author \\ Content \\ Introduction \\ 1. Chemical synthesis of polysilazanes \\ 2. The use of polysilazanes \\ 3. Compositions of polysilazanes \\ Consequence
}

Keywords: polyorganosilazanes, high-temperature ceramics. SiCN, NMR and IR spectroscopy.

\begin{abstract}
A review is given on the class of organosilicon compounds - polysilazanes. The review includes the history of the discovery of silazanes, the main chemical methods for producing silazanes, the main reactions taking place with the participation of silazanes, and the scope of application of silazanes. The review shows the composition of silazanes. The review consists of 40 literature sources.

The synthesis of polyorganosilazanes was first described in 1964 by Kruger and Rohov. In the interaction of ammonia with chlorosilanes (ammonolysis), trimeric or tetrameric cyclosilazanes were formed at the beginning and in the subsequent reaction at high temperatures with a catalyst to obtain polymers with a higher molecular weight. Ammonolysis of chlorosilanes is still the most important synthetic route to polysilazanes. The industrial production of chlorosilanes using the Muller-Roch process, first reported in 1940, served as the cornerstone for the development of silazane chemistry. In the 1960s, the first attempts to turn organosilicon polymers into quasiceramic materials were described. At this time, suitable ("pre-ceramic") polymers are heated to $1000{ }^{\circ} \mathrm{C}$. or higher. It was shown that the elimination of organic groups and hydrogen leads to a rearrangement of the molecular network with the formation of amorphous inorganic materials, which show unique chemical and physical properties. Using polymer-derived ceramics, new applications can be discovered, especially in the field of highstrength materials.
\end{abstract}

\section{References}

[1] https://ru.qwe.wiki/wiki/Polysilazane

[2] S.N. Borisov, M.G. Voronkov, E.Ya. Lukevits. Silicon organic elemental compounds. Leningrad: Chemistry. 1966. 544p. (russian)

[3] Yu.M. Varezhkin. Synthesis and application of polymers of cyclodisilazane structure. Survey information NIITEKHIM, Moscow. 1977. 145p. (russian)

[4] L.M. Khananashvili, K.A. Andrianov. Technology of elemental monomers and polymers. 2nd ed. Moscow: Chemistry. 1983. 400p. (russian)

[5] Salameh, Chrystelle; Bernard, Samuel; Gervais, Christel; Babonneau, Florence; Bruma, Alina; Malo, Sylvie; Miele, Philippe. Chemistry of a series of aluminum-modified polysilazanes. Synthesis, pyrolysis behaviour and microstructural evolution. Journal of the European Ceramic Society, Journal; Online Computer File. 2019. Vol.39. Iss.2-3. P.183-194.

[6] Method for the coupled production of trisilylamine and polysilazanes having a molar mass of up to $500 \mathrm{~g}$ /mol. Hoppe, Carl-Friedrich; Goetz, Christian; Uehlenbruck, Goswin; Rauleder, Hartwig. Dec 4, 2014. WO 2014191058. A1

[7] Method for preparing high-temperature-resistant thermoplastic organic polysialanes. Ma, Kechao; Yin, Hengbo; Hu, Yongming; Mao, Yulin; Wang, Aili; Feng, Yonghai; Shen, Lingqin; Xie, Wenjing. Dec 11, 2013. CN 103435810A

[8] Alkynyl-containing polysilazanes and preparation thereof. Hu, Jidong; Xie, Zemin; Zheng, Zhimin; Luo, Yongming; Zhang, Zhijie. Jun 22, 2005. CN 1629208A 
[9] Novel polysilazanes as precursors for silicon nitride/silicon carbide composites without "free" carbon. Hoerz, Markus; Zern, Achim; Berger, Frank; Haug, Joerg; Mueller, Klaus; Aldinger, Fritz; Weinmann, Markus. Journal of the European Ceramic Society. 2005. Vol.25. Iss.2-3. P.99-110. Journal 2004

[10] Synthesis of silazane compounds suitable for polymers used as adhesives or resin modifiers. Koo, SangWahn; Hann, Seung-Kyung; Shiba, Yasunari; Kim, Jung-Wo; Lee, Sang-Do. Mar 5, 2003. JP 2003064088A

[11] Synthesis and Characterization of Meltable and Soluble Reticulating Polysilazane Modified via Melamine Toward SiCN Ternary Ceramics. Zhao, Yangzhong; Shao, Changwei; Ji, Xiaoyu; Zhang, Shuai. Journal of Inorganic and Organometallic Polymers and Materials. 2020. Vol.30. Iss.6. P.20172026. Journal; Online.

[12] Manufacturing method of polysilazane using carbon dioxide solvent and polysilazane produced using the same. Park, In; Hwang, Ha Su; Lee, Ji Yeong. May 7, 2020. KR 2107744B1

[13] Process for preparing polysilazanes. Bossman, Stefan; Wang, Hongwan. Sep 13, 2018. WO 2018164709A1

[14] Synthesis of fluorine-modified polysilazanes via Si-H bond activation and their application as protective hydrophobic coatings. Furtat, P.; Lenz-Leite, M.; Ionescu, E.; Machado, R.A.F.; Motz, G. Journal of Materials Chemistry A: Materials for Energy and Sustainability. 2017. Vol.5. Iss.48. P.25509-25521. Journal; Online Computer File.

[15] Synthesis of novel aminophenylacetylene-capped polysilazane. Song, Ning; Ni, Lizhong; Zhou, Jian. Apr 16, 2014. CN 103724627A

[16] Anticorrosive heat-resistant polysilazane coating compositions. Harada, Shin, Jun 11, 1996. JP 08151538A

[17] The synthesis and pore property of hydrogen membranes derived from polysilazane as inorganic polymer. Kwon, Il Min; Song, In-Hyuck; Park, Young-Jo; Lee, Jae-Wook; Yun, Hui-Suk; Kim, HaiDoo. Journal of the Korean Ceramic Society. 2009. Vol.46. Iss.5. P.462-466.

[18] Research on chemical structures and properties of pre-ceramic polymer polyborosilazanes with different boron contents. Teng, Yadi; Huang, Xinlong, Gongneng Cailiao. Journal. 2016. Vol.47. P.5022-5029.

[19] Polymethyl (hydro)/polydimethylsilazane-derived coatings applied on AA1050: effect of the dilution in butyl acetate on the structural and electrochemical properties. Fedel, Michele; Rossi, Stefano; Deflorian, Flavio. Journal of Coatings Technology and Research. Journal; Online Computer File. 2019. Vol.16. Iss.4. P.1013-1019.

[20] Preparation and optical properties of Si-B-C-N bamboo nanomaterial from polymer. Wang, Yan-song; Xu, Shi-feng; Fan, Yi; Luo, Jin-song; Wang, Wen-quan; An, Li-nan; Zhang, Li-gong, Faguang Xueba. Journal. 2005. Vol.26. Iss.3. P.391-394.

[21] Polysilazanes. Soum, Alain, Silicon-Containing Polymers Conference. General Review. 2000. P.323-349.

[22] Antibacterial silicone rubber-based material for use to produce nipple. Xie, Liming. May 21. 2019. CN 109777122A

[23] Thermally insulating coatings based on polysilaxanes for the central cone of a steel piston. Dannenfeldt, Margrit; Meske, Ralf, Nov 8. 2018. WO 2018202861A1

[24] Synthesis of fluorine-modified polysilazanes via Si-H bond activation and their application as protective hydrophobic coatings. Furtat, P.; Lenz-Leite, M.; Ionescu, E.; Machado, R.A.F.; Motz, G. Journal of Materials Chemistry A: Materials for Energy and Sustainabilit. Journal; Online Computer File. 2017. Vol.5. Iss.48. P.25509-25521.

[25] Ambient-curable polysilazane coating compositions. Morikawa, Masashi; Koana, Tomoharu, Feb 22, 2000. JP 2000053920A

[26] Concrete parts with thermosetting polymer coating. Stigler, Martin; Langer, Otto; Rieder, Manfred; Liebl, Stefan, Apr 15, 2009. EP 2048125, A2

[27] Liquid glass useful as coating agents for iron-reinforced concrete. Saito, Hiroyuki; Handa, Takao; Matsuoka, Shigeto, Jun 30, 2011. JP 2011126723A

[28] Coating of steel parts for high oxidation resistance at elevated temperatures. Ujiie, Yoshinori; Nishiwaki, Kenichiro, Dec 27, 1993. JP 05345983A

[29] Preparation of yttrium-containing polysilazanes as starting material for preparation of yttriumcontaining composite powders and composite sintered bodies. Okabe, Masanori; Higuchi, Yoshikatsu; Kawakami, Yasunobu, Jul 12, 1995. EP 662463.A2

[30] Patent 20140349022, December 14, 2012, Date of publication: Nov 27, 2014, Inventor: Mark Nolde (St. Augustine, Florida) 
[31] Research of chemical structures and properties of pre-ceramic polymer polysilazanes with different compositions. Teng, Ya-di; Sun, Xiao-long; Huang, Xin-long; Sheng, Yong-gang; Zhou, Hua-feng, Gongneng Cailiao. Journal. 2015. Vol.46. Iss.7. P.7019-7024.

[32] Anti-adhesion coating composition. Li, Qiumu; Chang, Jun; Sun, Xiaowei; Wang, Fei, Apr 23, 2019. CN 109666403A

[33] Compositions for forming barrier layer, semiconductor substrate with barrier layer, method for producing substrate for solar cells, and method for manufacturing solar cell element. Orita, Akihiro; Yoshida, Masato; Nojiri, Takeshi; Kurata, Yasushi, Aug 21, 2014. WO 2014126117A1

[34] Compositions and process for preparing curable organofluorine-modified polysilazanes. Yang, Yu; Moore, George G. I.; Klun, Thomas P., Dec 4, 2012. US 8324324,B2

[35] US 8470924 B2 Color-pigmented paint composition having high covering power, increased scratch resistance, and easy-to-clean properties, Brand, Stefan; Dierdorf, Andreas; Liebe, Hubert; Osterod, Frank; Mahn, Markus; Ryan, Ken

[36] Use of polysilazanes as permanent anti-fingerprint coatings for metal surfaces, Brand, Stefan; Dierdorf, Andreas; Liebe, Hubert; Wacker, Andreas, Aug 31, 2006. WO 2006089649, A1

[37] Phosphorus-containing silazane composition with low dielectric constant for siliceous films, siliceous fillers, and semiconductor device. Aoki, Tomoko; Aoki, Hiroyuki. Jan 27, 2005. WO 2005007748A1

[38] Silicon carbonitride ceramics derived from polysilazanes Part I. Investigation of compositional and structural properties. Haluschka, Christoph; Kleebe, Hans-Joachim; Franke, Rainer; Riedel, Ralf. Journal of the European Ceramic Society. Journal. 2000. Vol.20. Iss.9. P.1355-1364.

[39] $R U 2374284$ C2, Published: 27.11.2009 No. 33, BRAND Stefan (DE), DIRDORF Andreas (DE), LIBE Hubert (DE), VACKER Andreas (DE), CLARIANT INTERNATIONL LTD (CH). (russian)

[40] US 8338351, Subbareddy Kanagasabapathy, Richard J. Baumgart, Wen-Chen Su, Frances E. Lockwood, Vinod Kanniah. 